\title{
Transmission Network Fault Location Observability With Minimal PMU Placement
}

\author{
Kai-Ping Lien, Student Member, IEEE, Chih-Wen Liu, Senior Member, IEEE, Chi-Shan Yu, Member, IEEE, and \\ Joe-Air Jiang, Member, IEEE
}

\begin{abstract}
This paper presents a concept of fault-location observability and a new fault-location scheme for transmission networks based on synchronized phasor measurement units (PMUs). Using the proposed scheme, minimal PMUs are installed in existing power transmission networks so that the fault, if it occurs, can be located correctly in the network. The scheme combines the fault-location algorithm and the fault-side selector. Extensive simulation results verify the proposed scheme.
\end{abstract}

Index Terms-Fault location, phasor measurement unit (PMU), transmission network.

\section{INTRODUCTION}

A CCURATE estimate of the fault location is critical to inspection, maintenance, and repair of transmission lines [1]-[8]. Several two-terminal fault-location algorithms based on phasor measurement units (PMUs) techniques have been proposed [9]-[13] recently. These PMU-based algorithms propose the calculation of fault location using synchronized voltage and current phasors. While they can achieve high accuracy in fault location, they are limited to locate faults in a transmission network installed by PMUs on every bus. Considering the installation cost of PMUs, it is important to investigate the placement scheme of the PMUs at minimal locations on the network in the sense that the fault-location observability can be achieved over the entire network. By borrowing from the concept of network state observability, which means that the entire steady-state bus voltage phasors can be estimated using the installed meter measurements, we define the fault-location observability as that if a fault occurs on the network, then it can be located exactly using the installed digital fault recorders such as PMUs.

Based on the topology of the power system, this paper first proposes a minimal placement strategy of PMUs, and then develops an innovative fault-location algorithm for the transmission network. Using installed PMUs, the new fault-location scheme can enhance the protection function of the network. In the proposed scheme, every line section in transmission networks only needs one-side PMU installation. The proposed

Manuscript received February 3, 2005; revised May 26, 2005. This work was supported by the National Science Council of Taiwan, R.O.C., under Contract NSC-93-2213-E-002-054. Paper no. TPWRD-00067-2005.

K.-P. Lien and C.-W. Liu are with the Department of Electrical Engineering, National Taiwan University, Taipei 106, Taiwan (e-mail: f89921115@ntu.edu.tw, cwliu@cc.ee.ntu.edu.tw).

C.-S. Yu is with the National Defense University, Chung-Cheng Institute of Technology, Taoyuan 335, Taiwan (e-mail: chsyu @ ccit.edu.tw).

J.-A. Jiang is with the Department of Bio-Industrial Mechatronics Engineering, National Taiwan University, Taipei 106, Taiwan, R.O.C. (e-mail: jajiang@ntu.edu.tw).

Digital Object Identifier 10.1109/TPWRD.2005.858806

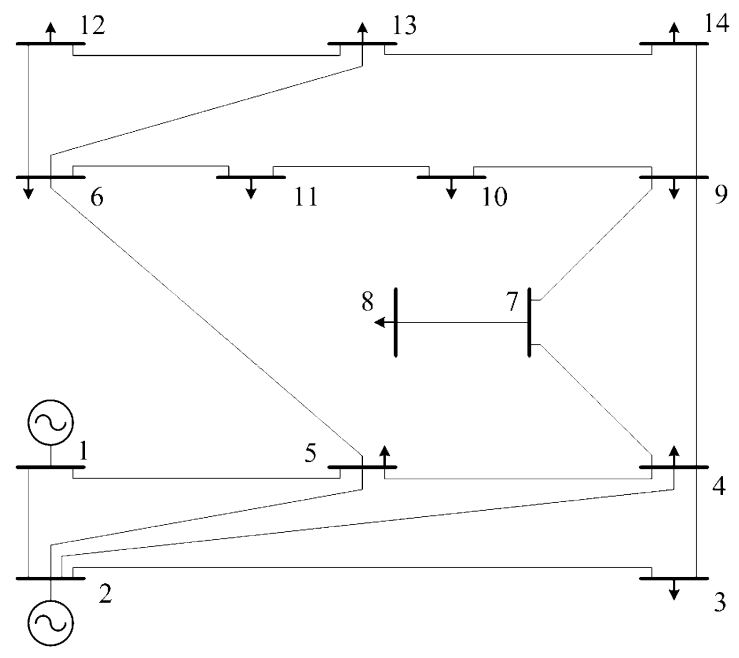

Fig. 1. IEEE-14 test buses.

fault-location scheme can accurately and quickly determine a fault point no matter where it occurs on the power grid. Meanwhile, the prefault transmission network models are not necessary in this approach. The proposed algorithm is more appropriate to practical applications.

\section{Minimal PMU Placement StRategy}

Based on the purpose of this work, this section will present a simple but minimal PMU placement strategy such that the faultlocation observability can be achieved. The PMUs placement proceeds with the following steps.

Step 1) PMUs are placed on the buses with the largest number of connected branches and with the second largest number of connected branches.

Step 2) PMUs are placeed on the buses by the so-called "one-bus spaced deployment strategy," which means that every two PMUs are spaced by one bus as possible as that the minimal PMUs are achieved and the proposed fault-location scheme can work.

Step 3) Prune PMUs deployment after the above two steps and eliminate the redundant PMUs until it violates the one-bus-spaced deployment strategy.

Notably, the selection of PMU's placement location is not unique in Step 2). The criterion for the proposed fault-location scheme can work properly in that every line section in the protected network needs at least one-side PMU installation.

We illustrate the placement strategy with Fig. 1, which is the IEEE 14-bus system. The sample network shown in Fig. 1 can 


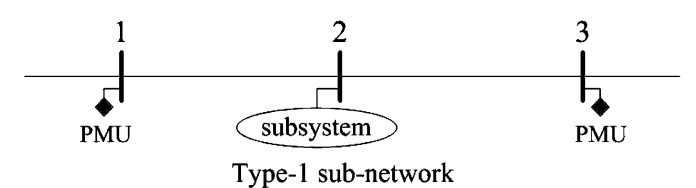

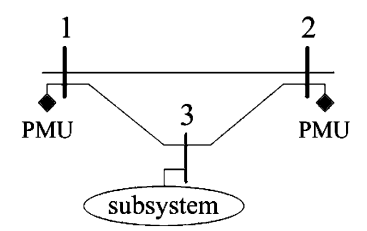

Type-2 sub-network

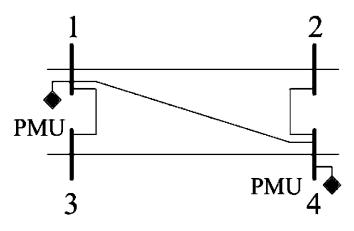

Type-4 sub-network

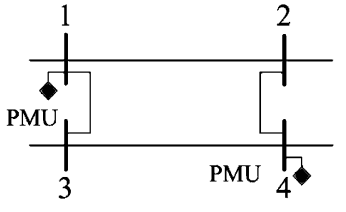

Type-3 sub-network

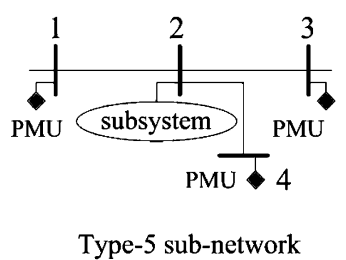

Type-5 sub-network
Fig. 2. Five subnetworks with one-bus-spaced deployment strategy.

be composed of five subnetworks, type- 1 to type- 5 as shown in Fig. 2. Each of the five subnetworks is placed with PMUs, which are called "one-bus-spaced deployment strategy." Indeed, most power transmission networks can be composed of these five subnetwork configurations. The type-1 subnetwork is the basic target of our fault-location algorithm developed in Section III. The fault-location algorithm for the type-1 subnetwork will be called the "one- bus-spaced fault-location scheme." The scheme can be directly applied to the fault-location calculation of type-2 to type-5 subnetworks. The reason is explained as follows.

In the type-2 subnetwork, since both ends of line 1-2 have been placed with PMUs, its fault-location task can be achieved using our previous two-terminal approach [9]. The configuration of line 1-3-2 of the type-2 subnetwork is the same with type-1. Also, the type-3 subnetwork can be viewed as a combination of two type-1 subnetworks connected in a parallel manner (i.e., line 1-2-4 and line 1-3-4). Therefore, a one-bus-spaced fault-location scheme can serve as the fault locator of these two cases.

As for type 4, except that there is one more line (i.e., line 1-4) than the type- 3 subnetwork, the other portion of the type- 4 subnetwork is the same with type-3. The faults in the type-4 subnetwork can also be located by the mentioned two-terminal approach [9] and one-bus-spaced fault-location scheme easily.

For type 5, finally, the lines 1-2-3, 1-2-4, or 3-2-4 are the same configuration of the type-1 subnetwork. Thus, if PMUs are placed on the buses 1,3 , and 4 , and the one-bus-spaced fault-location scheme is applied twice, the fault-location observability of the type- 5 subnetwork can be achieved. According to the proposed PMUs deployment strategy, any complicated transmission network can be divided into type- 1 to type- 5 subnetworks. Then, we apply the one-bus spaced fault-location scheme (proposed in Section III) and the two-terminal fault location algorithm developed in the previous paper [9] to the respective subnetworks. So, the fault-location observability can be achieved. As an example, we apply three steps to Fig. 1.

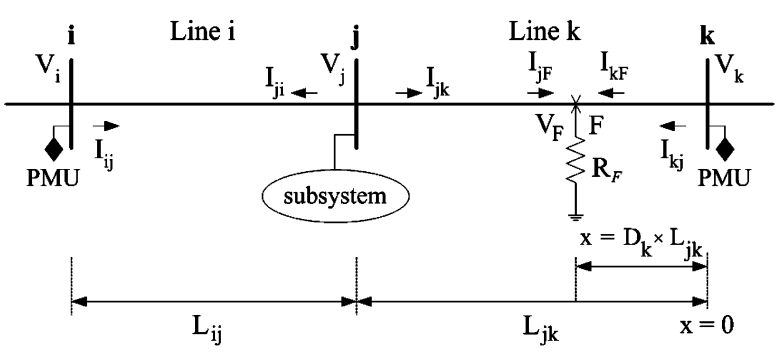

Fig. 3. Type-1 subnetwork with a fault.

First, according to Step 1), PMUs are placed on the buses 4, 5, 9, 2, and 6. Second, we place PMUs on buses 8, 10, and 13 according to one-bus-spaced deployment strategy. Finally, prune PMUs' deployment in the entire network and eliminate the redundant PMUs. As a result, only eight PMUs are needed to be placed on buses $2,4,5,6,8,9,10$, and 13 to achieve faultlocation observability. Notably, the placement of PMUs based on the proposed strategy is not unique. But the number of PMUs is minimal based on a one-bus-spaced deployment strategy.

In [14], the largest number of PMUs for power state observability is derived below

$$
v_{U B}=\left\lceil\frac{\left(N+\frac{m}{2}\right)}{3}\right\rceil
$$

where $N$ is the number of buses and $m$ is the number of unknown power injection. $\lceil x\rceil$ is an integer equal to or large than real number $x$.

For the illustrated sample system, $m$ is equal to 14 . So, it is found that, at most, seven PMUs should be installed to observe the operation state of the transmission network under normal conditions. Although, 8 PMUs from the proposed strategy are somewhat more than 7 PMUs placement for power state $n$ observability, the latter cannot locate the fault for transmission networks when a fault occurs.

\section{ONE-BUS-SPaced FAULT-LoCATION SCHEME}

Consider Fig. 3, which is the type-1 subnetwork with a fault. The PMUs are only installed in both bus $i$ and bus $k$. The type of subsystem connected to bus $j$ has no limitation. It can be generators, loads, or combined systems. Bus $j$ is connected at a distance of $L_{i j}$ away from bus $i$ and at a distance of $L_{j k}$ away from bus $k$, respectively. The distributed transmission line model is used to develop the fault-location algorithms.

Based on the measurement configuration, the fault-location algorithm for a transmission network can be developed. Fig. 4 illustrates the flowchart of the one-bus-spaced fault-location scheme for the sample network. The three-phase synchronized phasors of voltages and currents are measured simultaneously by PMU $i$ and PMU $k$. The phasor quantities are then decoupled into sequence components using symmetrical transformation and the symmetrical components are used as the input data for further computations. The fault-location algorithm developed here is based on symmetrical components of voltages and currents rather than directly using phase values since the former allows the three-phase system to be treated like three single circuits. By virtue of these decoupled single-phase circuits, 


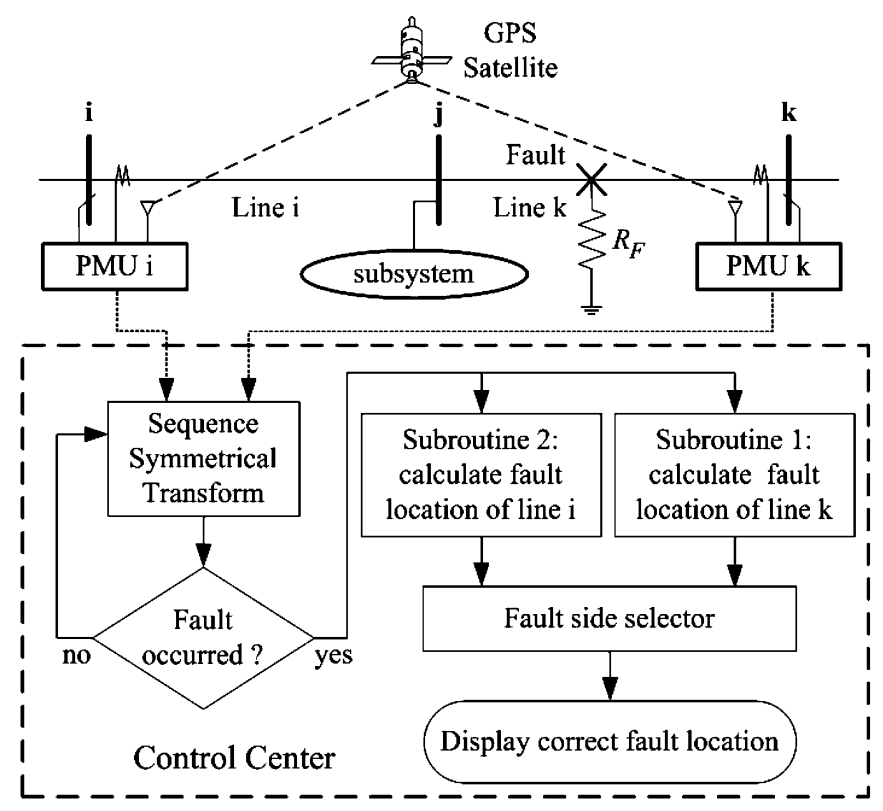

Fig. 4. Flowchart of the one-bus spaced fault-location scheme for the type-1 subnetwork.

the fault location of the original three-phase transmission line system can be also obtained and, thus, the computation burden is reduced significantly.

First, the fault detection indices from bus $i$ or bus $k$ can be used to trigger the proposed fault-location scheme from sleep state to activation state. Since the fault detector can not discriminate the true fault side between line $i$ and line $k$, two identical subroutines (subroutine1 and subroutine 2) are used to calculate the fault location in line $i$ and $k$, respectively. Then, the proposed fault-side selector is used to identify the correct fault side and fault location. Finally, the correct fault side and fault location are confirmed and displayed to operators.

The assumption of pure fault resistance [1], [2] is used to develop the proposed fault-location algorithm in subroutine 1 and subroutine 2 . We will show in our simulation results that the accuracy of the proposed fault-location algorithm will not be affected if the pure fault resistance assumption is violated. Since the fault-location algorithms in subroutine 1 and subroutine 2 are identical, only subroutine 1 is explained. The proposed fault-location algorithms and fault-side selector are developed as follows.

\section{A. Fault-Location Algorithm}

All variables in Fig. 3 can be thought of as symmetrical components vectors. As shown in Fig. 3, a fault labeled as $F$ is assumed to occur on line $k$ at a distance of $D_{k}$ (p.u.) away from bus $k$. When only bus $k$ is measured, the fault-location calculation of line $k$ is similar to that of the single-terminal fault location problems [1], [2]. However, the problem is more complex in this case, since the configuration of the subsystem is completely unknown.

In order to solve the fault location in line $k$, the measurements in bus $i$ are used to build the boundary conditions on bus $j$. First,

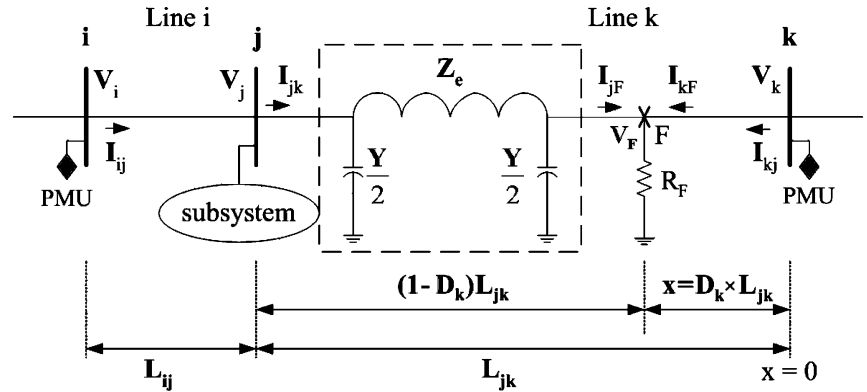

Fig. 5. Two-port hyperbolic $\pi$-model.

the transmission line between bus $j$ and the fault location $F$ is replaced by the two-port hyperbolic $\pi$-model depicted in Fig. 5. The variables in Fig. 5 are defined as follows:

$$
\begin{aligned}
& Z_{e}=Z_{c k} \sinh \left(r_{k}\left(1-D_{k}\right) L_{j k}\right) \\
& \frac{Y}{2}=\frac{1}{Z_{c k}} \tanh \left(r_{k}\left(1-D_{k}\right) L_{j k}\right)
\end{aligned}
$$

where $\left(1-D_{k}\right) L_{j k}$ (kilometers) is the line length of the $\pi$-model transmission line. $Z_{c k}$ and $r_{k}$ are the characteristic impedance and propagation constant of the transmission line $k$, respectively. The two-port $\pi$-network has four terminal variables $V_{j}$, $I_{j k}, V_{F}$, and $I_{j F}$. If we got any two of them as known variables, the remaining two unknowns can be easily solved via basic circuit computations [15].

Although bus $j$ is not measured, bus voltage $V_{j}$ can be calculated via the measurements at bus $i$ and is given in (3)

$$
V_{j}=\frac{\left(V_{i}+I_{i j} Z_{c i}\right)}{2 \exp \left(r_{i} L_{i j}\right)}+\frac{\left(V_{i}-I_{i j} Z_{c i}\right)}{2} \exp \left(r_{i} L_{i j}\right)
$$

where $Z_{c i}$ and $r_{i}$ are the characteristic impedance and propagation constant of the transmission line $i$, respectively. Thus, the left terminal voltage $V_{j}$ of the two-port $\pi$-network is known but the left terminal input current $I_{j k}$ is still unknown since the injection current of the subsystem is unknown. In addition, since the fault location is unknown, both the right terminal voltage and currents are also unknown variables. However, the right terminal voltage $V_{F}$ is only dependent on the unknown fault location $D_{k}$. If the fault location $D_{k}$ is obtained, the left terminal current $I_{j k}$ can also be obtained.

Therefore, there are two unknown variables $I_{j k}$ and $D_{k}$ left to be solved. Herein, the Gauss-Seidel numerical iteration method is used to solve the problem. According to the circuit theories and assumption constraint, the two groups of equations for two unknown variables are defined as

Group-1: Network equations

$$
F_{1}\left(I_{j k}, D_{k}, \theta\right)=0
$$

Group-2: Constraint equations

$$
F_{2}\left(I_{j k}, D_{k}, \theta\right)=0
$$

where $\theta$ represents the known variables, such as $V_{i}, V_{k}, V_{j}$, etc., and $I_{j k}$ and $D_{k}$ are the unknown variables. Group-1 equations are obtained from the two-port $\pi$-network enclosed by dashed 


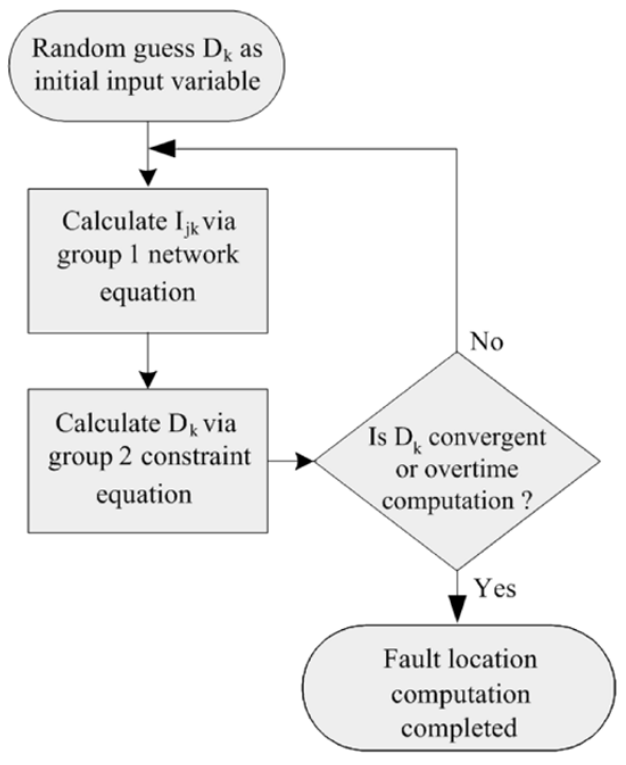

Fig. 6. Flowchart for the procedure of calculating $D_{k}$ and $I_{j k}$.

line in Fig. 5 to solve the unknown variable $I_{j k}$. Group-2 equations are obtained from the assumption of pure resistance fault impedance to solve the fault location $D_{k}$. Since the simultaneous equations of Group-1 and Group-2 are nonlinear, numerical method [16] is needed to find the solutions of $I_{j k}$ and $D_{k}$.

The calculation procedure is depicted in Fig. 6 and summarized as follows.

1) Given an initial value of $D_{k}$.

2) Substitute $D_{k}$ into Group-1 equations to calculate $I_{j k}$.

3) Substitute $I_{j k}$ into Group-2 equations to calculate $D_{k}$.

4) Repeat steps 2) and 3) until the final results of $D_{k}$ and $I_{j k}$ are obtained.

5) The Group-1 and Group-2 equations for different fault types are summarized in Appendix A.

Apply the same procedures to subroutine 2 computation; fault location $D_{i}$ (p.u.) away from bus $j$ can also be obtained.

\section{B. Fault-Side Selector}

Simultaneously applying the proposed fault-location computation to subroutines 1 and 2, two fault locations $D_{k}$ and $D_{i}$ can be obtained, respectively. Since only one is the correct result, a fault-side selector is developed to select the correct solution.

The resulting solutions of $D_{k}$ and $D_{i}$ in subroutines 1 and 2 can be classified into the following four conditions:

1) $D_{k} \in(0,1), D_{i} \notin(0,1)$;

2) $D_{k} \notin(0,1), D_{i} \in(0,1)$;

3) $D_{k} \in(0,1), D_{i} \in(0,1)$;

4) $D_{k} \notin(0,1), D_{i} \notin(0,1)$.

In order to obtain the correct solution, this work uses a technique proposed by Yu et al. [17]. In our extensive simulations and a simple proof given in Appendix B, we find that the calculated fault resistances $R_{F}$ of the correct and incorrect side are positive and negative, respectively. Moreover, since $D_{k}$ and $D_{i}$ are both per-unit representations of the fault location in line $k$ and line $i$, respectively, the correct $D_{k}$ or $D_{i}$ must obey the assumptions: $D_{k} \in(0,1)$ or $D_{i} \in(0,1)$. Thus, the correct solution in condition 1,2 , and 3 can be easily selected as $D_{k}$ and $D_{i}$,

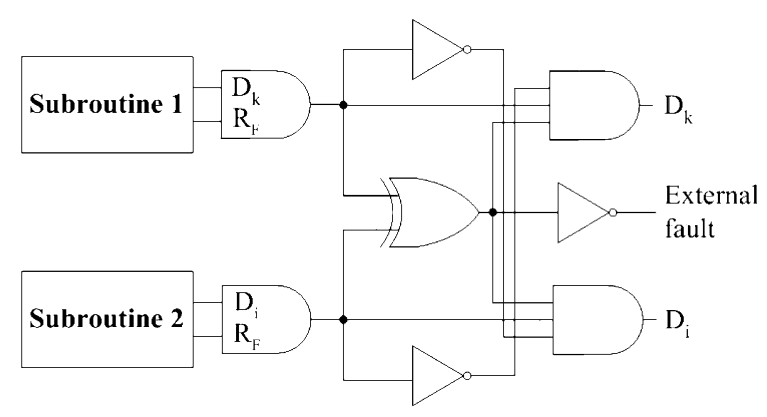

Fig. 7. Logic block of the proposed fault site selector.

respectively, by choosing corresponding positive $R_{F}$. If there is no corresponding positive $R_{F}$ for condition 1 to condition 3 , then the selector output is an external fault. As for condition 4, since the computed fault locations $D_{k}$ and $D_{i}$ do not fall into the interval $(0,1)$, the fault is identified as an external event.

From the above discussions, the fault-side selector can be easily implemented by the logic circuit shown in Fig. 7. In this case, let the logic signal of the computed fault locations $\left(D_{k}, D_{i}\right) \in(0,1)$ and positive $R_{F}$ be state "1;" otherwise, the logic signals are set to be be state "0." For example, when $\left(D_{k}, D_{i}\right) \notin(0,1)$, the two AND gates on the left-hand side of Fig. 7 will output logic "0" whatever $R_{F}$ values are computed. Then, the scheme classifies the fault as an external fault.

Note that the proposed fault-location algorithm uses the voltage and current measurements from both ends of the protected line even though only one PMU is placed at either end of the line. For example, suppose that a fault occurred at line $k$ is shown in Fig. 4. Although only one PMU $k$ is placed at Bus $k$ of Line $k$, the voltages and currents of the no-PMU Bus $j$ can still be estimated by the data provided from PMU $i$ at Bus $i$. This work can be achieved by applying two groups of equations: Group-1 network equations and Group-2 constraint equations. Therefore, the proposed method can be considered as a two-terminal-based fault-location algorithm. This means that the prefault load condition does not affect the fault-location accuracy of the proposed algorithm [18]. Therefore, it is not necessary to make any assumption, such as source impedance, source voltage, current distribution factor, etc., during the development of the proposed fault-location algorithm.

\section{Performance Evaluation}

Fig. 8 depicts a simulated $345-\mathrm{kV}$ transmission network system encountered in Taiwan. The EMTP/ATP simulator [19] is used to model the system and generate fault data to test the performance of the proposed algorithm. The sample system consists of eight transmission lines and eight buses. The transmission lines are modeled with distributed parameters. The related parameters of the simulated system are given in Table I.

According to the developed placement strategy of PMUs, three PMUs are placed on buses 2, 4, and 7 in the sample system. Three identified fault-location zones are Zone_1 (line 2 and line 3), Zone_2 (line 5 and line 6), and Zone_3 (line 7 and line 8), respectively. Lines 1 and 4 are out of the fault location. Thus, according to our previous discussion, the three 


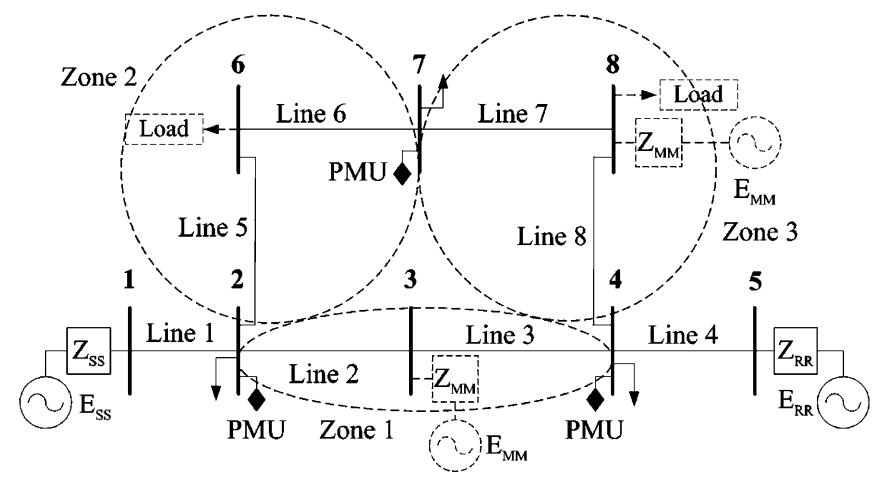

Fig. 8. Sample network for simulation tests. Depending on the need for tested cases, the equivalent voltage sources and loads behind buses 3,6 , and 8 were separately connected into the system.

TABLE I

PARAMETERS OF THE SIMULATION SYSTEM

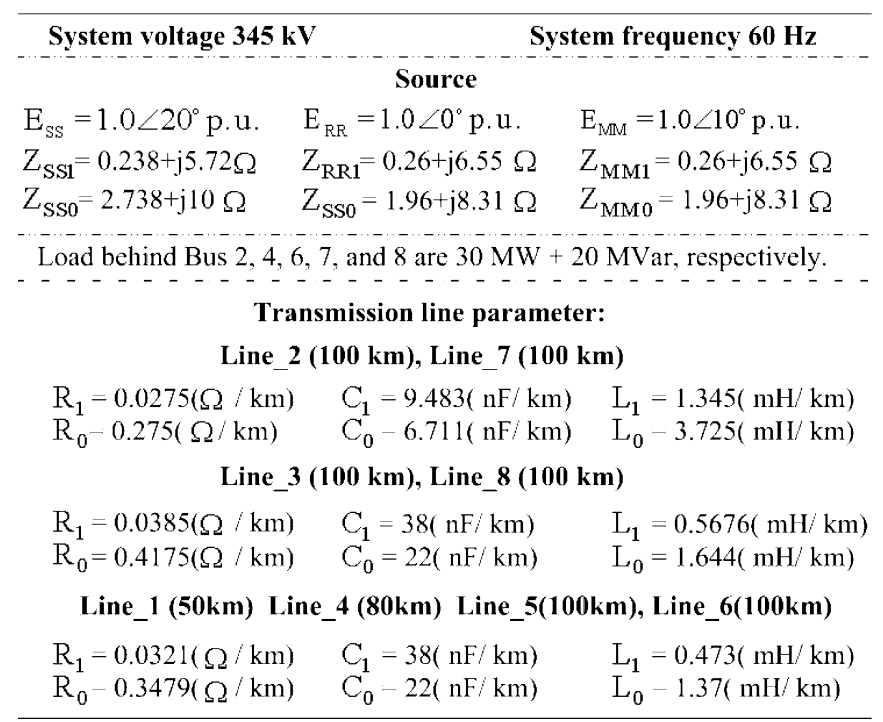

installed PMUs can provide fault-location observability for the transmission network area enclosed by buses $2,3,4,6,7$, and 8 .

This work uses a digital mimic filter [20] to eliminate dc offset and the phasors are estimated using the discrete Fourier transform (DFT) filtering algorithm working at 64 samples per cycle. The total simulation time is $100 \mathrm{~ms}$ and the percentage error of the estimated fault location is defined as

$$
\begin{aligned}
& \% \text { error } \\
& =\frac{\text { Estimated Location }- \text { Actual Location }}{\text { Total Line Length }} \times 100 \% \text {. }
\end{aligned}
$$

The fault locations, fault resistances, and the phase angles of fault currents are computed from subroutine 1 and subroutine 2 simultaneously.

To verify the performance of the proposed scheme, extensive simulation studies for various systems and fault conditions have been performed. The definitions for fault resistance locations in the multiphase transmission line are the same as those defined in [21] and [22]. Thus, for the ABCG fault, there are three identical fault resistances $R_{f}$ placed between the fault point of each phase and common point, respectively. Then, the common point is shorted to ground. For the ABS fault, there is one fault resistance $R_{f}$ placed between the fault points of A-phase and B-phase.
TABLE II

\begin{tabular}{|c|c|c|c|c|c|c|}
\hline Case & $\begin{array}{c}\text { Case description } \\
\mathrm{D}_{\mathrm{k}} \text { or } \mathrm{D}_{\mathrm{i}}: \text { p.u.; } \mathrm{R}_{\mathrm{F}}: \Omega\end{array}$ & $\begin{array}{l}\text { Subrou } \\
\mathrm{D}_{\mathrm{k}} \text { (p.u.) }\end{array}$ & $\begin{array}{l}\text { atine } 1 \\
\mathrm{R}_{\mathrm{F}}(\Omega)\end{array}$ & $\begin{array}{l}\text { Subrou } \\
\mathrm{D}_{\mathrm{i}} \text { (p.u.) }\end{array}$ & $\begin{array}{l}\text { utine } 2 \\
\mathrm{R}_{\mathrm{F}}(\Omega)\end{array}$ & $\begin{array}{l}\text { Selected } \\
\text { output }\end{array}$ \\
\hline 1 & Line $3, D_{k}=0.2, R_{F}=0.1, A G$ & 0.199 & 0.101 & 20.913 & 908.88 & $\mathbf{D}_{\mathbf{k}}$ \\
\hline 2 & Line $2, \mathrm{D}_{\mathrm{i}}=0.8, \mathrm{R}_{\mathrm{F}}=10, \mathrm{ABCG}$ & -8.218 & -28.75 & 0.801 & 10.020 & $\mathbf{D}_{\mathbf{i}}$ \\
\hline 3 & Line $6, D_{k}=0.3, R_{F}=10, B C G$ & 0.302 & 9.899 & 7.060 & -7369 & $\mathbf{D}_{\mathbf{k}}$ \\
\hline 4 & Line $5, D_{i}=0.6, R_{F}=0.1, A G$ & -13.21 & 105.27 & 0.601 & 0.113 & $\mathbf{D}_{\mathrm{i}}$ \\
\hline 5 & Line $7, D_{i}=0.8, R_{F}=10, A B S$ & 2.788 & 4884 & 0.798 & 10.117 & $\mathbf{D}_{\mathbf{i}}$ \\
\hline 6 & Line $8, D_{k}=0.9, R_{F}=10, A B C G$ & 0.897 & 10.928 & 0.874 & -173.3 & $\mathbf{D}_{\mathrm{k}}$ \\
\hline 7 & Line $1, D_{i}=1.1, R_{F}=100, A B C G$ & $\sim 0.74$ & $\sim-120$ & $\mathrm{X}$ & $\mathrm{X}$ & \# \\
\hline
\end{tabular}

Test Cases of the Proposed Fault-Side Selector

For the BCG fault, the fault points of B-phase and C-phase are shorted to a common point. Then, the common point is connected to the ground by one fault resistance $R_{f}$. Some results are presented and discussed below.

\section{A. Test for the Proposed Fault-Side Selector}

Extensive cases simulated by the EMTP/ATP simulator are utilized to examine the validity of the proposed fault-side selector. Some cases are briefly described in the second column of Table II. In case 2, for example, a three-line-to-ground fault occurs at $80 \mathrm{~km}$ (i.e., $D_{i}=0.8$ p.u.) in line 2 away from bus 3 . The fault resistance of this simulation case is assumed to be $10 \Omega$. In the tested cases 1 and 2, a generator modeled as a voltage source $E_{\mathrm{MM}}$ is connected behind Bus 3. In cases 3 and 4, a load modeled as $30 \mathrm{MW}+21 \mathrm{MVar}$ at nominal voltage is connected behind Bus 6 . In cases 5 and 6, a generator and load are connected behind Bus 8 . The load is modeled as $30 \mathrm{MW}+21 \mathrm{MVar}$ at nominal voltage and the generator is modeled as a voltage source $E_{\mathrm{MM}}$. Case 7 is a three-phase-grounded fault in line 1 with a distance of $10 \mathrm{~km}$ away from Bus 2, in which the grounded resistance is set as $100 \omega$. Cases $1-5$ belong to condition 1 or 2 of the proposed fault-side selector mentioned in Section III. Case 6 is the simulated cases for conditions 3 of the fault-side selector. The simulation results are summarized in the remaining portion of Table II.

For case 6, Fig. 9(a) and (b) illustrate the calculation responses of fault-location indices (i.e., $D_{k}$ and $D_{i}$ ), and fault resistance $R_{F}$ calculated by subroutines 1 and 2, respectively. The inspection of these responses reveals that both of the calculated location indices $D_{k}$ and $D_{i}$ enter soon into the setting range of condition 3 (i.e., $D_{k}$ and $D_{i} \in(0,1)$ ). In this case, the proposed fault-side selector can automatically take the calculated $R_{F}$ and the phase angles of fault currents into consideration to select the correct fault location. In this case, the correct fault location is easily selected as $D_{k}=0.897$ (p.u.) which has an estimated positive fault resistance and the fault location error is $0.251 \%$. Case 7 is an external fault case in line 1. The fault event is a three-phase ground fault in line 1 which is $10 \mathrm{~km}$ away from Bus 2 . The fault resistance is $100 \omega$. Fig. 10(a) and (b) show the responses of fault-location indices (i.e., $D_{k}$ and $D_{i}$, and fault resistance $R_{F}$ calculated by subroutines 1 and 2, respectively). An examination of the shown responses indicates that case 7 belongs to condition 1. And, the fault-side selector will output an external fault, 

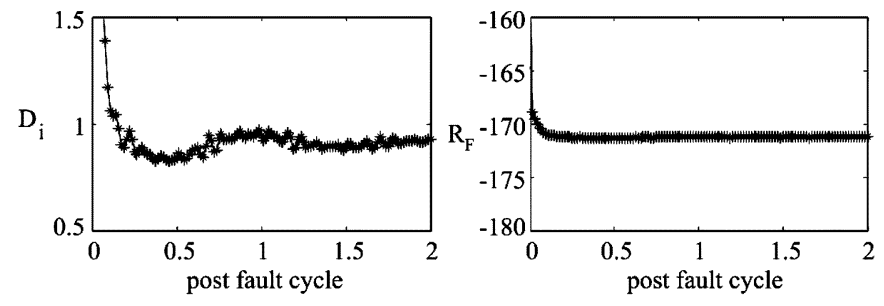

(a) Plots of $\mathrm{D}_{\mathrm{i}}$ and $\mathrm{R}_{\mathrm{F}}$ calculated by subroutine 2
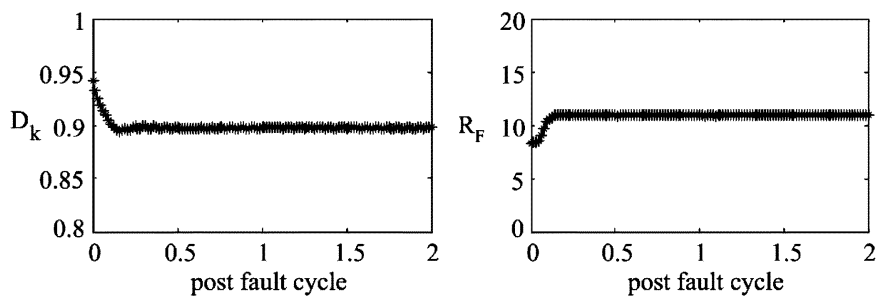

(b) Plots of $D_{k}$ and $R_{F}$ calculated by subroutine 1

Fig. 9. Responses of fault-location indices and fault resistance calculated by subroutine 1 and 2 for simulation case 6 .
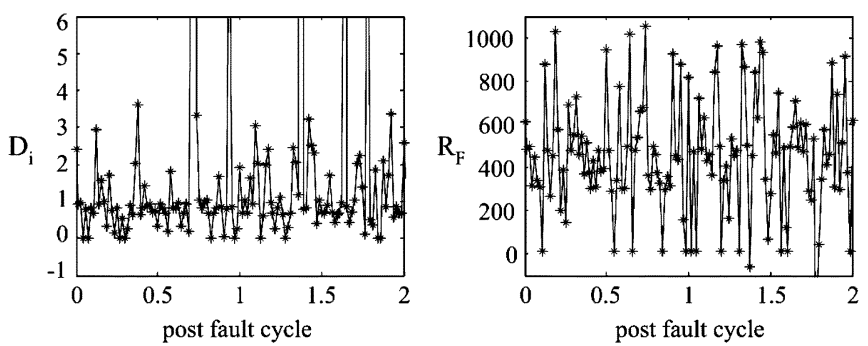

(a) Plots of $\mathrm{D}_{\mathrm{i}}$ and $\mathrm{R}_{\mathrm{F}}$ calculated by subroutine 2
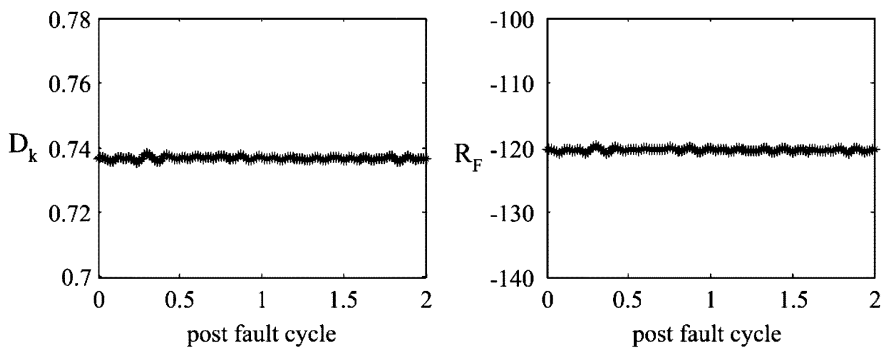

(b) Plots of $D_{k}$ and $R_{F}$ calculated by subroutine 1

Fig. 10. Responses of fault-location indices and fault resistance calculated by subroutine 1 and 2 for simulation case 7 .

because $R_{F}$ is negative for $D_{k}$. From Table II, it is clearly seen that the proposed fault-side selector determines the correct fault location.

\section{B. Statistical Evaluation}

To verify the robustness of the proposed scheme, extensive fault simulations under various system operations and fault events were conducted. This test considers different fault types, resistances, locations, and inception angles as statistical tests. Any test case could be one of the combinations of the following fault conditions:

- fault locations: $0.1,0.25,0.5,0.75$, and 0.9 p.u. of the line length in every tested line of the entire sample system;

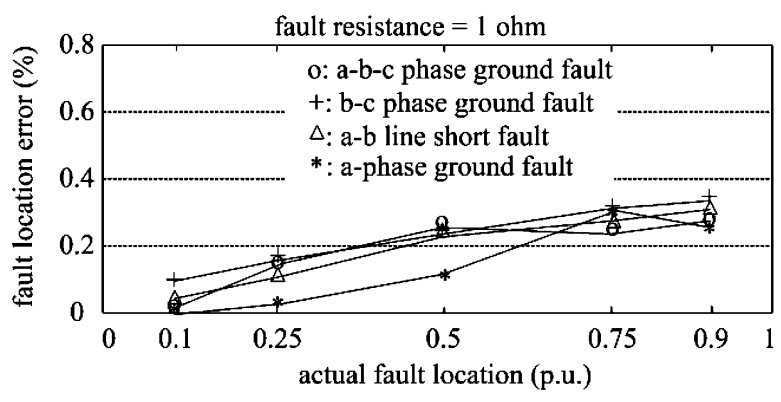

Fig. 11. Statistical evaluation of the fault-location estimation with respect to different faults away from Bus 7 (receiving end) occurred in line 6.

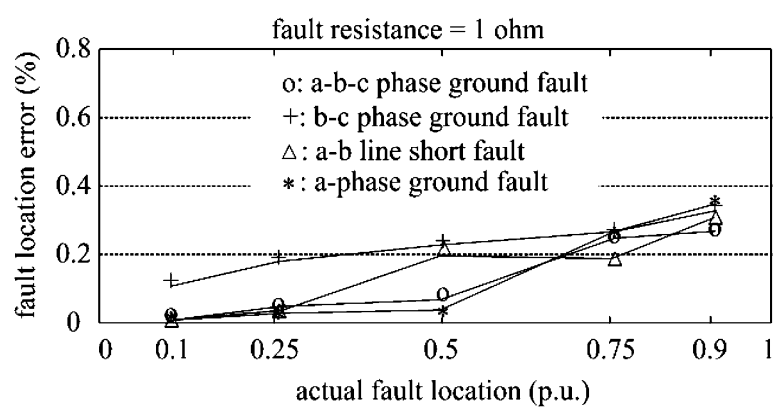

Fig. 12. Statistical evaluation of fault-location estimation with respect to different faults away from Bus 4 (receiving end) occurred in line 8.

- fault types: A-G, A-B-S, A-B-G, B-C-G, A-B-C-G, etc., where letters $\mathrm{S}$ and $\mathrm{G}$ represent short circuit and ground, respectively;

- fault resistance: $0.1,0.5,1,5,10$, and $100 \omega$;

- fault inception angles: $0^{\circ}, 45^{\circ}, 90^{\circ}, 135^{\circ}$, and $180^{\circ}$ in relation to the zero cross of a-phase voltage at the receiving end bus of the considered line.

- tested lines: line 1-line 8, in which line 1 and line 4 are not included in the protection zone.

The statistical evaluations of different faults in line 6 and 8 are selected to be shown in Figs. 11 and 12, respectively. In these simulated cases, all of the fault-location errors for every tested fault location have been expressed as the average errors of the mentioned inception angles. From the figures, it is clearly seen that the fault-location error is relatively insensitive to the fault types. The maximum error usually occurs in the remote ends [i.e., bus 6 and 8 (the uninstalled PMU bus)], and the errors for the entire tested cases were much smaller than $0.5 \%$.

Fig. 13 illustrates the impacts of different fault resistances under different fault distances in line 2. An examination of Fig. 13 clearly reveals that the proposed algorithm is hardly affected by the magnitudes of fault resistance. Because the proposed scheme does not involve the fault resistance, it is practically independent of fault resistance.

A total of more than 1800 simulation cases have been conducted with respect to various system situations and fault events for the entire sample networks. The statistical evaluations of the fault-location errors and the failure cases for the proposed algorithm under different fault resistances are briefly summarized in Table III. 


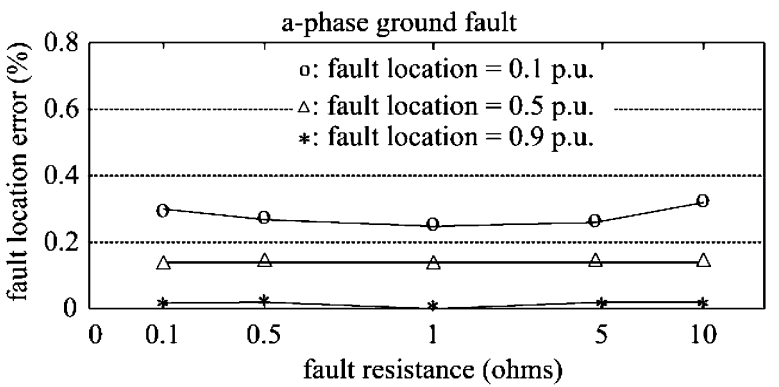

Fig. 13. Effects of faults resistances on the fault-location estimation away from Bus 3 (receiving end) occurred in line 2.

TABLE III

SUMMARY FOR THE STATISTICAL EVALUATIONS OF THE PROPOSED FAULT-LOCATION ALGORITHM

\begin{tabular}{|c|c|c|c|c|c|}
\hline Line & $\begin{array}{l}\text { Total tested cases } \\
\mathrm{R}_{\mathrm{F}}=0.1,1,10 \Omega\end{array}$ & $\begin{array}{l}\text { Failed } \\
\text { cases }\end{array}$ & $\begin{array}{c}\text { Error } \\
(\%)\end{array}$ & $\begin{array}{l}\text { Total tested cases } \\
\mathrm{R}_{\mathrm{F}}=100 \Omega\end{array}$ & $\begin{array}{l}\text { Failed } \\
\text { cases }\end{array}$ \\
\hline $1^{*}$ & 40 & 0 & -- & 10 & 0 \\
\hline 2 & 250 & 0 & 0.159 & 50 & 2 \\
\hline 3 & 250 & 0 & 0.187 & 50 & 6 \\
\hline 4* & 40 & 0 & -- & 10 & 0 \\
\hline 5 & 250 & 0 & 0.184 & 50 & 4 \\
\hline 6 & 250 & 0 & 0.177 & 50 & 1 \\
\hline 7 & 250 & 0 & 0.145 & 50 & 1 \\
\hline 8 & 250 & 0 & 0.162 & 50 & 3 \\
\hline
\end{tabular}

* Lines 1 and 4 are out of the protection zone.

The scheme correctly detects all of the fault events with the fault resistance of less than $100 \omega$ and provides very good accuracy in fault location. When the fault resistance is even up to $100 \omega$, only a few fault events cannot be detected by the proposed approach. The failure rate of this statistical study is very low, about $17 / 1800=0.94 \%$. In all cases, the maximum error is $0.637 \%$ and the average error is about $0.169 \%$.

\section{Effects of Nonpure Fault Resistance}

As in [1] and [2], our proposed fault-location algorithms also assume the fault impedance is a pure resistance $R_{f}\left(R_{f}\right.$ is placed on the fault path) and uses the assumption to establish the constraint equations in Appendix A. However, if the fault impedance is not pure resistance, we must investigate and estimate the impact of fault impedance on the proposed fault-location algorithm. To answer this question, we have conducted many simulations. For example, assume that a different type fault occurs in the location of 0.25 p.u. of line 2 in the sample system shown in Fig. 8. The original fault resistance is set to be $1 \omega$. Then, let the magnitude of the imaginary part of a fault impedance be increased gradually. When X/R is tuned to be $10 \%$, the error of the fault location is just about $0.01 \%$ as shown in Table IV. The result is almost the same as the condition of pure resistances. Even though the ratio of $\mathrm{X} / \mathrm{R}$ is increased to $50 \%$, the fault-location error is only about $2.83 \%$. Except the above-mentioned example, extensive simulations with respect to various ground faults, including single-phase to ground faults, also show similar results. Thus, it can be concluded that the impact of nonpure resistance of the fault path on the proposed algorithm is very slight.
TABLE IV

SUMMARY FOR THE IMPACT OF FAULT IMPEDANCE VARIATIONS ON THE PERFORMANCE OF THE PROPOSED FAULT-LoCATION ALGORITHM

\begin{tabular}{|c|c|c|c|c|}
\hline \multirow{2}{*}{ Case* } & \multirow{2}{*}{$\mathrm{R}_{\mathrm{F}}(\Omega)$} & \multicolumn{2}{|c|}{ Calculated results } & \multirow{2}{*}{$\begin{array}{c}\text { Location } \\
\text { Error (\%) }\end{array}$} \\
\hline & & $\mathrm{D}$ (p.u.) & $\mathrm{R}_{\mathrm{F}}(\Omega)$ & \\
\hline 1 & 1 & 0.2499 & 1 & 0.01 \\
\hline 2 & $1+\mathrm{j} 0.02$ & 0.2499 & 1.0002 & 0.01 \\
\hline 3 & $1+j 0.04$ & 0.2499 & 1.0008 & 0.01 \\
\hline 4 & $1+j 0.06$ & 0.2499 & 1.0018 & 0.01 \\
\hline 5 & $1+j 0.08$ & 0.2499 & 1.0032 & 0.01 \\
\hline 6 & $1+\mathrm{j} 0.1$ & 0.2499 & 1.005 & 0.01 \\
\hline 7 & $1+j 0.3$ & 0.2412 & 1.047 & 0.88 \\
\hline 8 & $1+\mathrm{j} 0.5$ & 0.2217 & 1.129 & 2.83 \\
\hline
\end{tabular}

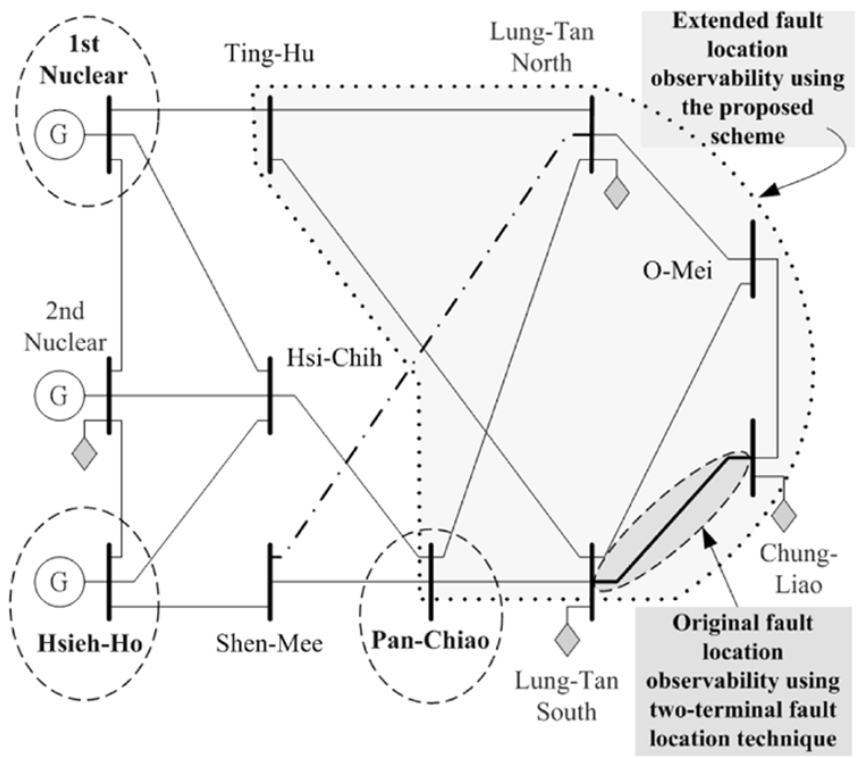

Fig. 14. Status of PMU deployment in the middle-to-north portion of the TPC transmission network system.

\section{Realistic Example}

In Taiwan, the phasor measurement system was employed in Taiwan Power Company (TPC) [23]. The extra-high-voltage [(EHV), 345-kV] transmission network of TPC includes 22 substations. Presently, eight PMUs were installed at eight EHV substations and mainly used an online phasor monitoring system and fault locators for the transmission lines. For example, Fig. 14 depicts the PMU deployment in the middle-to-north portion of the TPC transmission line system, in which four PMUs have been placed on the buses of Lung-Tan North, 2nd Nuclear, Chung-Liao, and Lung-Tan South, respectively. However, due to the limited installation of PMUs, only the transmission line between Chung-Liao and Lung-Tan South buses encircled by a dashed line can use our previous two-terminal fault-location technique. All of the other transmission lines cannot be applied by the existing fault-location technique. In order to enlarge the fault-location observability, the fault-location scheme proposed in this paper can be applied 
TABLE V

SUMMARY FOR GROUP EQUATIONS OF SUBROUTINE 1

\begin{tabular}{|c|c|c|}
\hline \multirow{2}{*}{$\begin{array}{c}\text { Group I: } \\
\text { Network Equation ( } \mathrm{n}=0,1,2) \\
\mathrm{V}_{\mathrm{Fn}}=\frac{\left(\mathrm{V}_{\mathrm{kn}}+\mathrm{I}_{\mathrm{kjn}} Z_{\mathrm{ckn}}\right)}{2} \mathrm{e}^{\left(\mathrm{r}_{\mathrm{kn}} \mathrm{D}_{\mathrm{k}} \mathrm{L}_{\mathrm{jk}}\right)} \\
+\frac{\left(\mathrm{V}_{\mathrm{kn}}-\mathrm{I}_{\mathrm{kjn}} Z_{\mathrm{ckn}}\right)}{2} \mathrm{e}^{\left(-\mathrm{r}_{\mathrm{kn}} \mathrm{D}_{\mathrm{k}} \mathrm{L}_{\mathrm{jk}}\right)}\end{array}$} & \multicolumn{2}{|r|}{$\begin{array}{c}\text { Group 2: } \\
\text { Constraint Equation }\end{array}$} \\
\hline & $\begin{array}{l}\mathrm{a}-\mathrm{g} \\
\text { fault }\end{array}$ & $\begin{array}{l}\operatorname{Re}\left\{\mathrm{I}_{\mathrm{FO}}+\mathrm{I}_{\mathrm{F} 1}+\mathrm{I}_{\mathrm{F} 2}\right\} \\
\times \operatorname{Im}\left\{\mathrm{V}_{\mathrm{F} 0}+\mathrm{V}_{\mathrm{F} 1}+\mathrm{V}_{\mathrm{F} 2}\right\} \\
=\operatorname{Re}\left\{\mathrm{V}_{\mathrm{FO}}+\mathrm{V}_{\mathrm{F} 1}+\mathrm{V}_{\mathrm{F} 2}\right\} \\
\times \operatorname{Im}\left\{\mathrm{I}_{\mathrm{FO}}+\mathrm{I}_{\mathrm{F} 1}+\mathrm{I}_{\mathrm{F} 2}\right\}\end{array}$ \\
\hline $\begin{array}{l}I_{j k n}=\frac{\left(V_{j n}-V_{F_{n}}\right)}{Z_{e}}+V_{j n} \times \frac{Y}{2} \\
I_{F n}=\frac{\left(V_{k n}+I_{k j n} Z_{c k n}\right)}{2} e^{\left(r_{k n} D_{k} L_{j k}\right)}\end{array}$ & & $\begin{array}{l}\operatorname{Re}\left\{\mathrm{I}_{\mathrm{F} 1}-\mathrm{I}_{\mathrm{F} 2}\right\} \\
\times \operatorname{Im}\left\{\mathrm{V}_{\mathrm{F} 1}-\mathrm{V}_{\mathrm{F} 2}\right\} \\
=\operatorname{Re}\left\{\mathrm{V}_{\mathrm{F} 1}-\mathrm{V}_{\mathrm{F} 2}\right\} \\
\times \operatorname{Im}\left\{\mathrm{I}_{\mathrm{F} 1}-\mathrm{I}_{\mathrm{F} 2}\right\}\end{array}$ \\
\hline$+\frac{\left(\mathrm{V}_{\mathrm{kn}}-\mathrm{I}_{\mathrm{kjn}} Z_{\mathrm{ckn}}\right)}{2} \mathrm{e}^{\left(-\mathrm{r}_{\mathrm{kn}} \mathrm{D}_{\mathrm{k}} \mathrm{L}_{\mathrm{jk}}\right) / Z_{\mathrm{ckn}}}$ & $\begin{array}{l}b-c-g \\
\text { fault }\end{array}$ & $V_{F 1}=V_{F 2}$ \\
\hline $\begin{array}{l}+\frac{\left(v_{j n}+1_{j k n} Z_{c k n}\right)}{2 \exp \left(r_{k n} L_{R}\right)} e^{\left(r_{k n} D_{k} L_{j k}\right)} \\
+\frac{\left(V_{k n}-I_{k j n} Z_{c k 1}\right)}{2 \exp \left(r_{k n} L_{k}\right)} e^{\left(-r_{k n} D_{k} L_{j k}\right) / Z_{c k n}}\end{array}$ & $\begin{array}{l}\text { a-b-c-g } \\
\text { fault }\end{array}$ & $\begin{array}{l}\operatorname{Re}\left\{\mathrm{I}_{\mathrm{F} 1}\right\} \times \operatorname{Im}\left\{\mathrm{V}_{\mathrm{F} 1}\right\} \\
=\operatorname{Re}\left\{\mathrm{V}_{\mathrm{F} 1}\right\} \times \operatorname{Im}\left\{\mathrm{I}_{\mathrm{F} 1}\right\}\end{array}$ \\
\hline
\end{tabular}

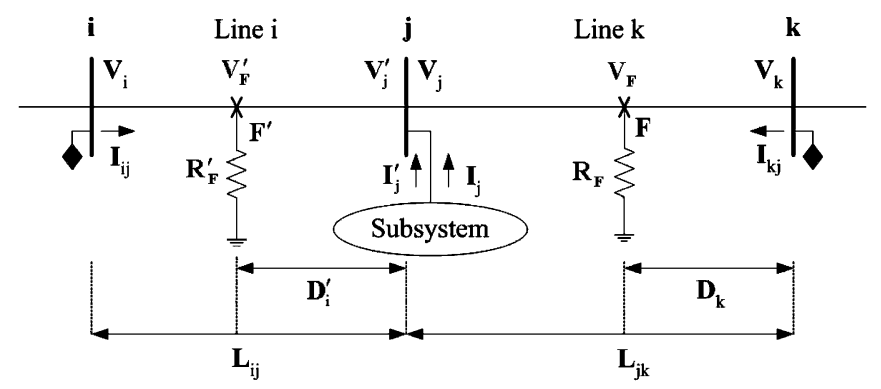

Fig. 15. One-line diagram for the demonstration of selecting an algorithm.

to the present system. As a result, the fault-location observability can be enlarged to the area encircled by dotted line except the line between Shen-Mee and Lung-Tan North buses. Notably, there is no extra installation of PMUs. Moreover, using the proposed scheme, only 3 PMUs should be added on the buses Hsieh-Ho, 1st Nuclear, and Pan-Chiao, and the fault-location observability can be achieved over the entire middle-to-north TPC transmission network.

\section{CONCLUSION}

This work has successfully developed a new fault-location scheme for transmission networks using PMUs. The proposed fault-location scheme is economic in the sense that minimal PMUs are used. This paper presents a concept of fault-location observability. The scheme combines the fault-location algorithm and the fault-side selector. Extensive simulation results verify the proposed scheme.

\section{APPENDIX A}

\section{GROUP EQUATIONS OF SUBROUTINES 1 AND 2}

The group equations of subroutine 1 for different types of faults are derived and summarized in Table V. All variables in Table $\mathrm{V}$ are the same as those defined in Fig. 3. The variables with subscript 0,1 , and 2 denote the 0,1 , and 2 sequence variables, respectively. The group equations of subroutine 2 also can be derived using the same ideas in Table V.

\section{APPENDIX B \\ SiMPLE PROOF OF THE FAULT-SIDE SELECTOR}

Herein, the proposed selector is proved via the short distance transmission-lines model shown in Fig. 15. The subsystem connected behind bus $j$ can be a generator or load. The fault occurs at transmission line $k$. The fault location $D_{k}$ (p.u.) is the correct fault location and the fault location $D_{i}^{\prime}$ (p.u.) is assumed to be the incorrect fault location. Meanwhile, the fault location $D_{k}$ and $D_{i}^{\prime}$ are on transmission lines $k$ and $i$, respectively.

Using KVL, the relations of $V_{k}$ and $V_{i}$ are obtained as follows:

$$
\begin{aligned}
V_{k} & =V_{j}-\left(I_{i j}+I_{j}\right)\left(1-D_{k}\right) L_{j k} Z_{k}+I_{k j} D_{k} L_{j k} Z_{k} \\
V_{j} & =V_{i}-I_{i j} L_{i j} Z_{i} \\
V_{k} & =V_{j}^{\prime}+I_{k j} L_{j k} Z_{k} \\
V_{j}^{\prime} & =V_{i}-I_{i j}\left(I-D_{i}^{\prime}\right) L_{i j} Z_{i}+\left(I_{j}^{\prime}+I_{k j}\right) D_{i}^{\prime} L_{i j} Z_{i} .
\end{aligned}
$$

From (B.1) and (B.2), the following relation is obtained:

$$
\begin{aligned}
\left(I_{i j}+I_{j}+I_{k j}\right) L_{j k} Z_{k} & -\left(I_{i j}+I_{j}+I_{k j}\right) D_{k} L_{j k} Z_{k} \\
& +\left(I_{i j}+I_{j}^{\prime}+I_{k j}\right) D_{i}^{\prime} L_{i j} Z_{i}=0
\end{aligned}
$$

where $\left(I_{i j}+I_{j}+I_{k j}\right)=I_{F}$ is the fault current of the correct side, and $\left(I_{i j}+I_{j}^{\prime}+I_{k j}\right)=I_{F}^{\prime}$ is the fault current of the incorrect side.

Rewrite (B.3) as

$$
\left(1-D_{k}\right) I_{F} L_{j k} Z_{k}+D_{i}^{\prime} I_{F}^{\prime} L_{i j} Z_{i}=0
$$

where $\left(1-D_{k}\right) L_{j k}$ and $D_{i}^{\prime} L_{i j}$ are all positive and real constants, $Z_{k}$ and $Z_{i}$ are per-unit-length impedances of transmission lines $k$ and $i$, and can be expressed as $Z_{k}=\left|Z_{k}\right| \angle \alpha^{\circ}$ and $Z_{i}=$ $\left|Z_{i}\right| \angle \beta^{\circ}$, respectively.

Rewrite (B.4) as

$$
\begin{aligned}
\frac{I_{F}}{I_{F}^{\prime}} & =-\frac{D_{i}^{\prime} L_{i j} Z_{i}}{\left(1-D_{k}\right) L_{j k} Z_{k}} \\
& =-\frac{D_{i}^{\prime} L_{i j}\left|Z_{i}\right| \angle \beta^{\circ}}{\left(1-D_{k}\right) L_{j k}\left|Z_{k}\right| \angle \alpha^{\circ}} \\
& =-K \angle\left(\beta^{\circ}-\alpha^{\circ}\right) .
\end{aligned}
$$

Since $K$ is positive and real constant, the phase difference between $I_{F}$ and $I_{F}^{\prime}$ must be $180^{\circ}+\beta^{\circ}-\alpha^{\circ}$. Normally, $\mid \beta^{\circ}-$ $\alpha^{\circ} \mid<90^{\circ}$. So, the phase difference between $I_{F}$ and $I_{F}^{\prime}$ must be $90^{\circ} \sim 270^{\circ}$. Meanwhile, due to the pure resistance assumption of the fault impedance, if $R_{F} R_{F}^{\prime}>0$ or $R_{F}^{\prime}>0$, the phase difference between $V_{F}$ and $V_{F}^{\prime}$ must be $90^{\circ} \sim 270^{\circ}$. From conventional circuit theory, a phase difference of $90^{\circ} \sim 270^{\circ}$ between $V_{F}$ and $V_{F}^{\prime}$ is impossible, since the phase difference between the sending end and receiving end is much less than $90^{\circ}$. If $R_{F} R_{F}^{\prime}<0$ or $R_{F}^{\prime}<0$ (since $R_{F}$ is positive), the phase difference between $V_{F}$ and $V_{F}^{\prime}$ must be $-90^{\circ} \sim 90^{\circ}$. A phase difference of $-90^{\circ} \sim 90^{\circ}$ between $V_{F}$ and $V_{F}^{\prime}$ is possible, since the phase difference between sending end and receiving end is much less than $90^{\circ}$. The possible phase difference between $V_{F}$ and $V_{F}^{\prime}$ 
is less than $90^{\circ}$. Thus, the estimated fault location with positive fault resistance is selected as the correct solution. When the fault occurs at line $i$, the same results can be obtained by the similar procedures demonstrated before.

\section{REFERENCES}

[1] L. Eriksson, M. M. Saha, and G. D. Rockefeller, "An accurate fault locator with compensation for apparent reactance in the fault resistance resulting from remote-end infeed," IEEE Trans. Power App. Syst., vol. PAS-104, no. 2, pp. 424-436, Feb. 1985.

[2] T. T. Takagi, Y. Yamakoshi, J. Baba, K. Uemura, and T. Sakaguchi, "Development of a new fault locator using the one-terminal voltage and current data," IEEE Trans. Power App. Syst., vol. PAS-101, no. 8, pp. 2892-2898, Aug. 1982.

[3] A. G. Phadke, "Synchronized phasor measurements in power systems," IEEE Comput. Appl. Power, vol. 6, no. 2, pp. 10-15, Apr. 1993.

[4] R. K. Aggarwal, D. V. Doury, A. T. Johns, and A. Kalam, "A practical approach to accurate fault location on extra high voltage teed feeder," IEEE Trans. Power Del., vol. 8, no. 3, pp. 874-883, Jul. 1993.

[5] D. G. Hart, D. Novosol, and E. Udren, "Application of synchronized phasors to fault location analysis," in Applications of Synchronized Phasors Conf., Precise Measurements in Power Systems Arlington, VA, Oct. 1993, vol. III-6.1-13.

[6] A. A. Girgis, D. G. Hart, and W. L. Peterson, "A new fault location technique for two- and three-terminal lines," IEEE Trans. Power Del., vol. 7, no. 1, pp. 98-107, Jan. 1992.

[7] M. Kezunovic, J. Mrkic, and B. Perunicic, "An accurate fault location algorithm using synchronized sampling," Elect. Power Syst. Res., vol. 29, no. 3, pp. 161-169, May 1994.

[8] A. O. Ibe and B. J. Cory, "A traveling wave based fault locator for twoand three-terminal networks," IEEE Trans. Power Syst., vol. PWRD-1, no. 1, pp. 283-288, Jan. 1986.

[9] J.-A. Jiang, J.-Z. Yang, Y.-H. Lin, C.-W. Liu, and J.-C. Ma, “An adaptive PMU based fault detection/location technique for transmission lines-part I: Theory and algorithms," IEEE Trans. Power Del., vol. 15, no. 2, pp. 486-493, Apr. 2000.

[10] J.-A. Jiang, Y.-H. Lin, J.-Z. Yang, T.-M. Too, and C.-W. Liu, "An adaptive PMU based fault detection/location technique for transmission lines-part II: PMU implementation and performance evaluation," IEEE Trans. Power Del., vol. 15, no. 4, pp. 1136-1146, Oct. 2000.

[11] C.-S. Chen, C.-W. Liu, and J.-A. Jiang, "A new adaptive PMU based protection scheme for transposed/untransposed parallel transmission lines," IEEE Trans. Power Del., vol. 17, no. 2, pp. 395-404, Apr. 2002.

[12] C.-S. Yu, C.-W. Liu, S.-L. Yu, and J.-A. Jiang, "A new PMU based fault location algorithm for series compensated lines," IEEE Trans. Power Del., vol. 17, no. 1, pp. 33-46, Jan. 2002.

[13] Y.-H. Lin, C.-W. Liu, and C.-S. Yu, "A new fault locator for three-terminal transmission line-using two-terminal synchronized voltage and current phasors," IEEE Trans. Power Del., vol. 17, no. 2, pp. 452-459, Apr. 2002.

[14] T. L. Baldwin, L. Mili, M. B. Boisen Jr, and R. Adapa, "Power system observability with minimal phasor measurement placement," IEEE Trans. Power Syst., vol. 8, no. 2, pp. 707-715, May 1993.

[15] C. A. Gross, Power System Analysis. New York: Wiley, 1986.

[16] J. R. Rice, Numerical Methods, Software, and Analysis. San Diego, CA: Academic, 1993.

[17] C.-S Yu, C.-W Liu, and Y.-H Lin, "A fault location algorithm for transmission lines with tapped leg-PMU based approach," in Proc. Power Eng. Soc. Summer Meeting, vol. 2, Jul. 2001, pp. 915-920.
[18] D. Novosel, D. G. Hart, E. Udren, and M. M. Saha, "Fault location using digital relay data," IEEE Comput. Appl. Power, vol. 8, no. 3, pp. 45-50, Jul. 1995.

[19] H. Dommel, "Electromagnetic Transient Program," BPA, Portland, OR, 1986.

[20] G. Benmouyal, "Removal of DC-Offset in current waveforms using digital mimic filtering," IEEE Trans. Power Del., vol. 10, no. 2, pp. 621-630, Apr. 1995.

[21] W. D. Stevenson Jr, Elements of Power System Analysis. New York: McGraw-Hill, 1982.

[22] W. A. Elmore, Protective Relaying Theory and Applications. Coral Springs, FL: ABB, 1994.

[23] C.-W Liu, "Phasor measurement applications in Taiwan," in Proc. IEEE Transmission and Distribution Conf. Exhibit., vol. 1, Yokohama, Japan, Oct. 2002, pp. 490-493.

Kai-Ping Lien (S'01) was born in Tainan, Taiwan, R.O.C., in 1970. He received the B.S. degree in electrical engineering from National Sun Yat-Sen University, Kaoshuing, in 1992. He is currently pursuing the Ph.D. degree in electrical engineering at National Taiwan University, Taipei.

Chih-Wen Liu (S'93-M'96-SM'02) was born in Taiwan, R.O.C., in 1964. He received the B.S. degree in electrical engineering from National Taiwan University (NTU), Taipei, Taiwan, in 1987, and the M.S. and Ph.D. degrees in electrical engineering from Cornell University, Ithaca, NY, in 1992 and 1994, respectively.

Currently, he is with NTU, where he is a Professor of electrical engineering. His main research interests include the application of computer technology to power system monitoring, protection, and control. His research interests include motor control and power electronics.

Chi-Shan Yu (S'99-M'01) was born in Taipei, Taiwan, R.O.C., in 1966. He received the B.S. and M.S. degrees in electrical engineering from National Tsing Hua University, Hsinchu, in 1988 and 1990, respectively, and the Ph.D. degree in electrical engineering from National Taiwan University, Taipei, in 2001.

Currently, he is Associate Professor of Electrical Engineering with the National Defense University, Chung-Cheng Institute of Technology. His research areas are computer relaying, power system transient stability controller design, and power electronics.

Joe-Air Jiang (M'01) was born in Tainei, Taiwan, R.O.C., in 1963. He received the M.S. and Ph.D. degrees in electrical engineering from National Taiwan University, Taipei, in 1990 and 1999, respectively.

Currently, he is an Associate Professor of bio-industrial mechatronics engineering at National Taiwan University. His research interests are in computer relaying, mechatronics, and bioeffects of the electromagnetic wave. 